\title{
USING Hybrid Adaptive Techniques to Reduce Multipath Effects in S-PCN Mobile Terminals
}

\author{
Sunday E. Iwasokun, Michael O. Kolawole \\ Department of Electrical and Electronics Engineering, School of Engineering and Engineering \\ Technology, Federal University of Technology, Akure, Nigeria \\ E-mail:wasokunday@yahoo.com \\ Received April 24, 2010; revised June, 16, 2010; accepted August 2, 2010
}

\begin{abstract}
Multipath signal processing is a promising technique for increasing the capacity of downlink frequency of satellite communication networks (S-PCN). The paper presents an approach to processing and reducing multipath signals received from S-PCN typified of mobile terminal users in clustered or mountainous environment. Use of hybrid linear adaptive antenna array technique and adaptive filtering technique provides improved performance by eliminating uncorrelated signal residing in antenna sidelobes.
\end{abstract}

Keywords: Multipath Signal, S-PCN, Hybrid Adaptive Technique, Adaptive Antenna Array, Mobile Terminal

\section{Introduction}

Satellite personal communication networks (S-PCN) has been improved and touted to provide communication networks to vast region of the earth. S-PCN faces multiple of challenges particularly those related to user terminals as well as the space segment regulatory challenges [1], and technical issues including topography and multiple access [2], link diversity and traffic allocation [3], and delayed signal component [4]. S-PCNs are subject to multipath propagation caused by scattering from objects in the vicinity of the satellite footprints and mobile terminal. In mountainous terrain, the mountain may be visible (line-of-sight) to both the satellite and the handheld terminal and act as large reflectors.

Some channels in S-PCN have one or more clusters of propagation paths for which each cluster has large number of paths with small differential delays. Some of these multipath signals may act as interference to one or more of better signal components; implying that for each cluster, the differences among the path delays are small on the adaptive antenna array. In any case, the position given by a satellite-based system relies on propagation time measurement. Hence, the environment of the antenna will play a major role because the propagation phenomena caused by the environment induce indirect propagation paths that introduce delays [5].

In this paper, the alternate path (multipath) reception is used as the only major signal available on mobile terminal. Most mobile stations transceivers are ideally circularly polarized and isotropic in nature, with a single low gain antenna element. However, these single element units are particularly more susceptible to noise interference because they receive signals from all directions. The resolution for a channel in which the multipath arises as a result of specular reflections off a number of objects and each individual multipath component is not dispersed in time [6]. The output of a specular multipath channel consists of the sum of a number of attenuated time-delayed versions of the transmitted signal from satellite, each of which arrives at the mobile terminal without distortion [7]. The adaptive antenna has been recognized as way to enhance capacity and coverage of the system [8]. Adaptive antenna array mounted on the mobile terminal is an approach suitable to combating unwanted signals in the sidelobes of antenna pattern as communication satellite traverses the sky, which this study attempts to investigate.

In some cases, line-of-sight (direct) and multipath (indirect) signals may be available to handheld terminal, the use of switched antenna diversity may assist the handheld terminal to determine which of the signals received is stronger in term of transmitted power component, and then to switch to this antenna for a given time interval [9]. In the case where there is no line-of-sight signal from the satellite but numerous multipath signals might be inci- 
dent on the handheld terminal unit, the antenna with the strongest incident signal will be selected to receive the signal.

\section{Array Geometry}

An adaptive array antenna unit is designed, as in Figure 1, bringing all the signals received by the various elements from a particular source into phase for further processing, as well as achieving some desired performance, such as maximizing the received signal-to-noise ratio (SNR). Drawing from [10] and [11], we analyse the antenna array assuming that 1 ) there is a direct path for the signal from the satellite and interfering signal is uncorrelated with the desired signal, 2) all the array elements are placed closely enough such that there is no significant amplitude variation due to the difference in propagation path length for any two elements, and 3) there is no significant difference in $\theta$ - the direction-of-arrival (DOA) - of a particular plane wave at any two elements.
For $\mathrm{N}$ elements array of identical characteristics, the first pulse is taken as the phase centre, $f(t-\tau)$. The second pulse is advanced by $\tau$ and denoted by $f(t-\tau)$, the third pulse is advanced by $2 \tau$ and denoted by $f(t-2 \tau)$, and so on. For convenience, five elements are used, so

$$
\begin{aligned}
f_{T}(t)= & a_{o} f(t)+a_{1} f(t-\tau)+a_{2} f(t-2 \tau)+ \\
& a_{3} f(t-3 \tau)+a_{4} f(t-4 \tau)
\end{aligned}
$$

where $a_{0}, a_{1} \ldots a_{5}$ are the weights/gains of each element of the array. In adaptive antenna array, the weights/gains of each element are taken to be the same (i.e., $\left.a_{0}=a_{1}=\ldots=a_{4}\right)$, conforming to practical situation, and the elements to be equally spaced. Had the weights variable, there is possibility they may modulate the desired signal. Now, if the first pulse is Fourier transformed and is represented by $f_{\Delta}(\omega)$, then

$$
\begin{aligned}
f_{T}(\omega)= & a_{0} f_{\Delta}(\omega)+a_{1} f_{\Delta}(\omega) e^{-j \omega \tau}+a_{2} f_{\Delta}(\omega) e^{-j 2 \omega \tau}+ \\
& a_{3} f_{\Delta}(\omega) e^{-j 3 \omega \tau}+a_{4} f_{\Delta}(\omega) e^{-j 4 \omega \tau}
\end{aligned}
$$

By multiplying (2) by $e^{-j \omega \tau}$ and then subtracting the

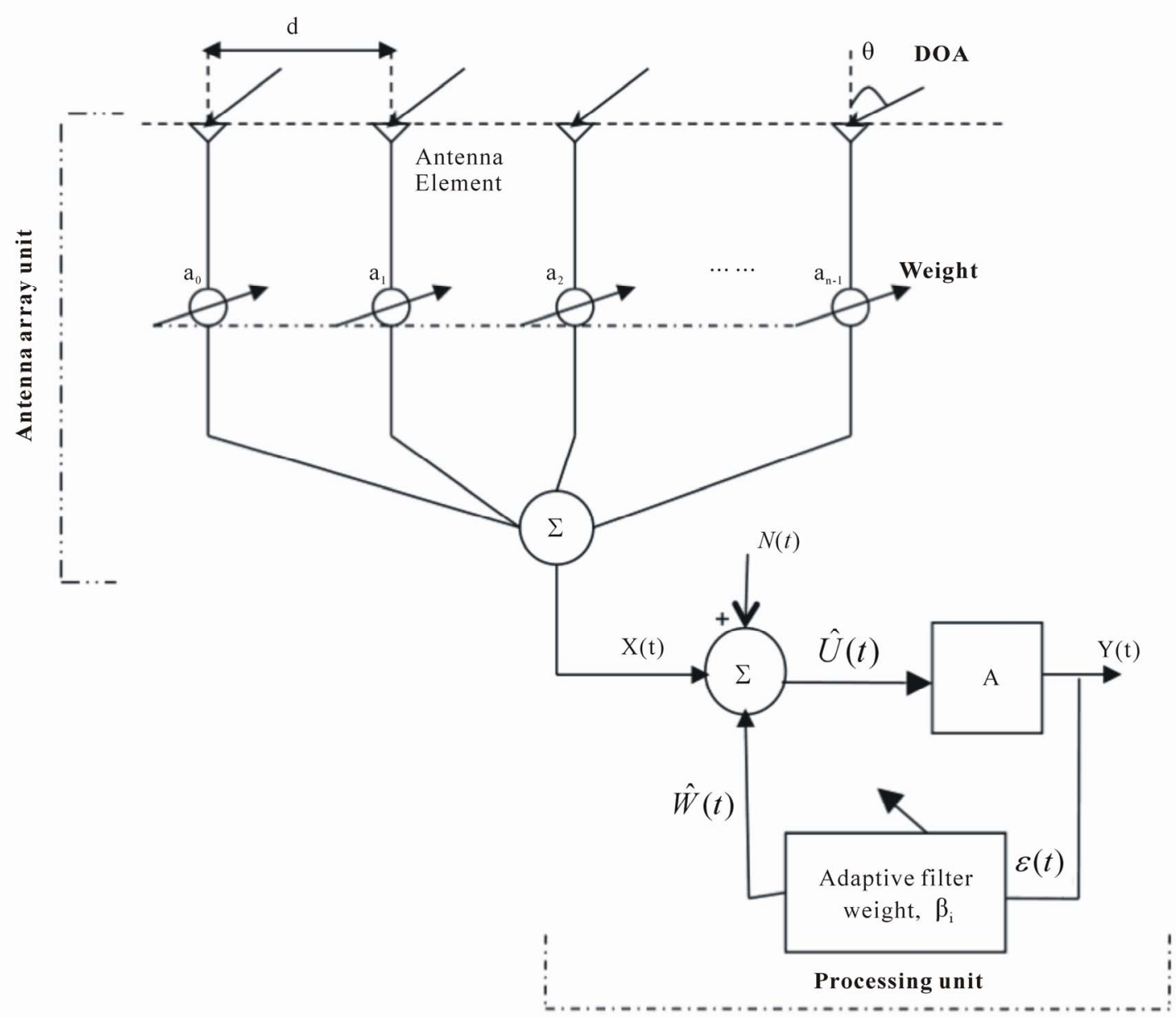

Figure 1. Hybrid adaptive processing system for S-PCN mobile terminal. 
resulting expression from (1), and rearranging ensuing expression and using known geometric series expansion we have

$$
f_{\tau}(\omega)=a_{0} f_{\Delta}(\omega)\left[e^{-j 2 \omega \tau}\left(\frac{\sin (5 \pi f \tau)}{\sin (\pi f \tau)}\right)\right]
$$

Typical S-PCN frequencies of 1.376 and $1.80 \mathrm{GHz}$ [12, 13] are used to examine the antenna array's behaviour using (3). Resulting graphs are shown in Figure 2. The sidelobes performance has an important impact on interference received from adjacent satellites (and ground based signals operating on same bands), as well as in determining antenna noise temperature. To successfully eliminate, or reduce significantly, the effect of external interference, the sidelobes have to be attenuated, if not removed. The sidelobes are cancelled or removed in this paper by the adaptive array process.

\section{Processing Unit: Modelling Interferences with Adaptive Filter}

Our approach models the effect of interferers on the S-PCN systems using interferences as recursive random processes, $N_{i}(t)$ with the array antenna. The input to the processor in Figure $\mathbf{1}$ is the inverse Fourier $\mathfrak{J}^{-1}\left[f_{\tau}(\omega)\right]$ of the array output, which now becomes $X(t)$ to the summer. The effect of noise from each antenna element is factored in at the output; that is, for $n$ elements with equal average signal-to-noise (SNR), the average SNR of the array combiner is $10 \log _{10}(n)$, which is higher in $\mathrm{dB}$ than the average SNR of any one of the elements [14]. Prior to processing, unit-step delay is introduced into the incoming signals and then superimpose on the signal of interest. The superposition allows the system model of the handheld unit to determine which of the arriving signals is stronger, and then switch to the antenna for a given time interval before filtration to eliminate or reduce interference.

Following Figure 1, the recursive-filtering algorithm can be represented as a linear, discrete-time $(t)$ model of the form:

$$
\begin{gathered}
\hat{U}(t)=X(t)+N(t)-\hat{W}(t) \\
Y(t)=A \hat{U}(t)
\end{gathered}
$$

where $A(t)$ is the system parameter; $\hat{W}(t)$ is optimum adaptive but weighted filter and $N(t)$ is additive, uncorrelated system interference, assumed white, zeromean Gaussian and stochastic; and $Y(t)$ filter (or measurement) output. The optimum interference $\hat{W}(t)$ is defined as

$$
\hat{W}(t)=\sum_{i=0}^{k} \beta_{i}(t) N_{t h}(t-i)
$$

where $N_{\text {th }}$ is the acceptable interference threshold, and $k$ is the filter order. The proceeding coefficient of the filter can be estimated from the present coefficient and other thresholds [15]:

$$
\beta_{i}(t+1)=\beta_{i}(t)+2 \eta X(t) N_{t h}(t-i)
$$

where $N_{t h}$ is the convergence constant.

The adaptive filter adapts the filter coefficients to achieve desired signal ensuring convergence; that is,

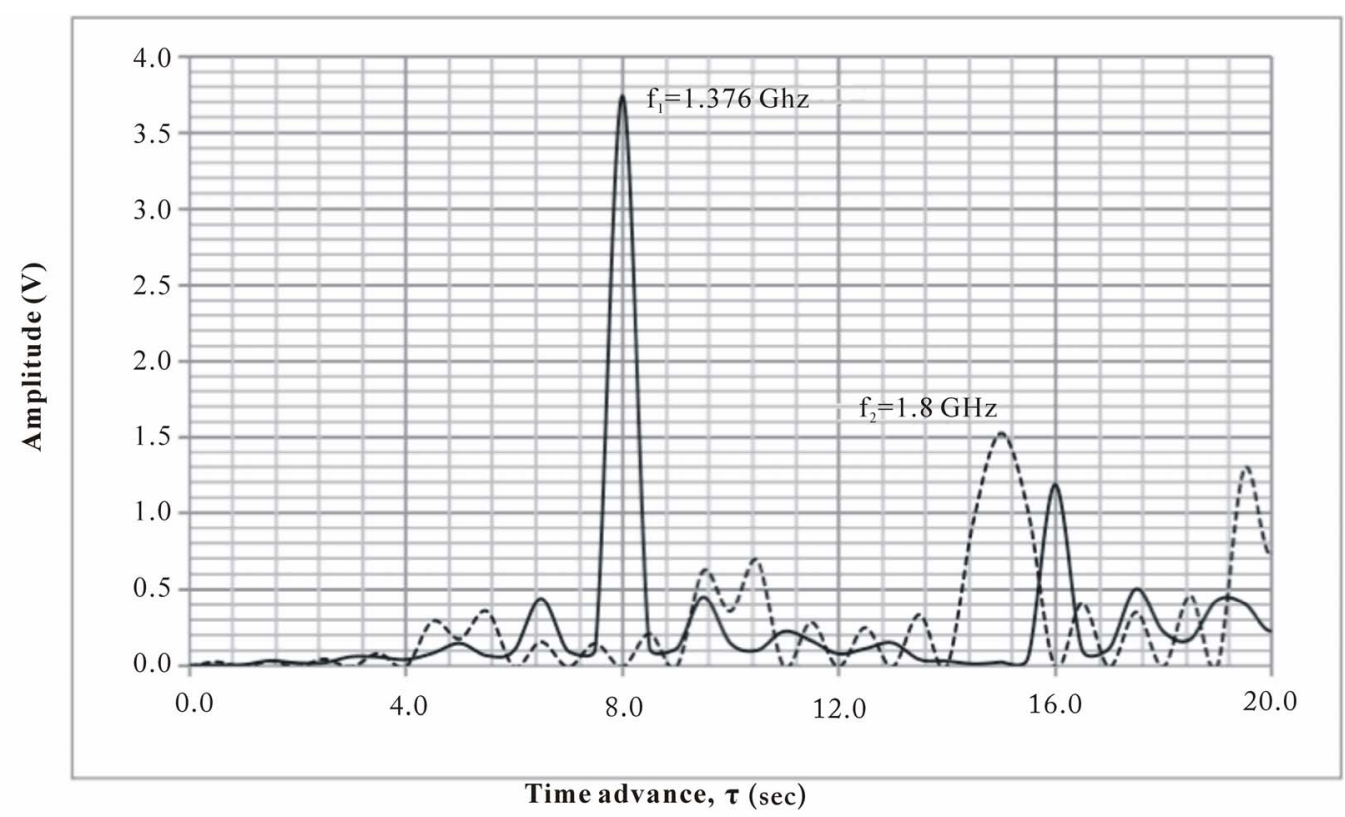

Figure 2. Antenna array's response at typical S-PCN frequencies. 


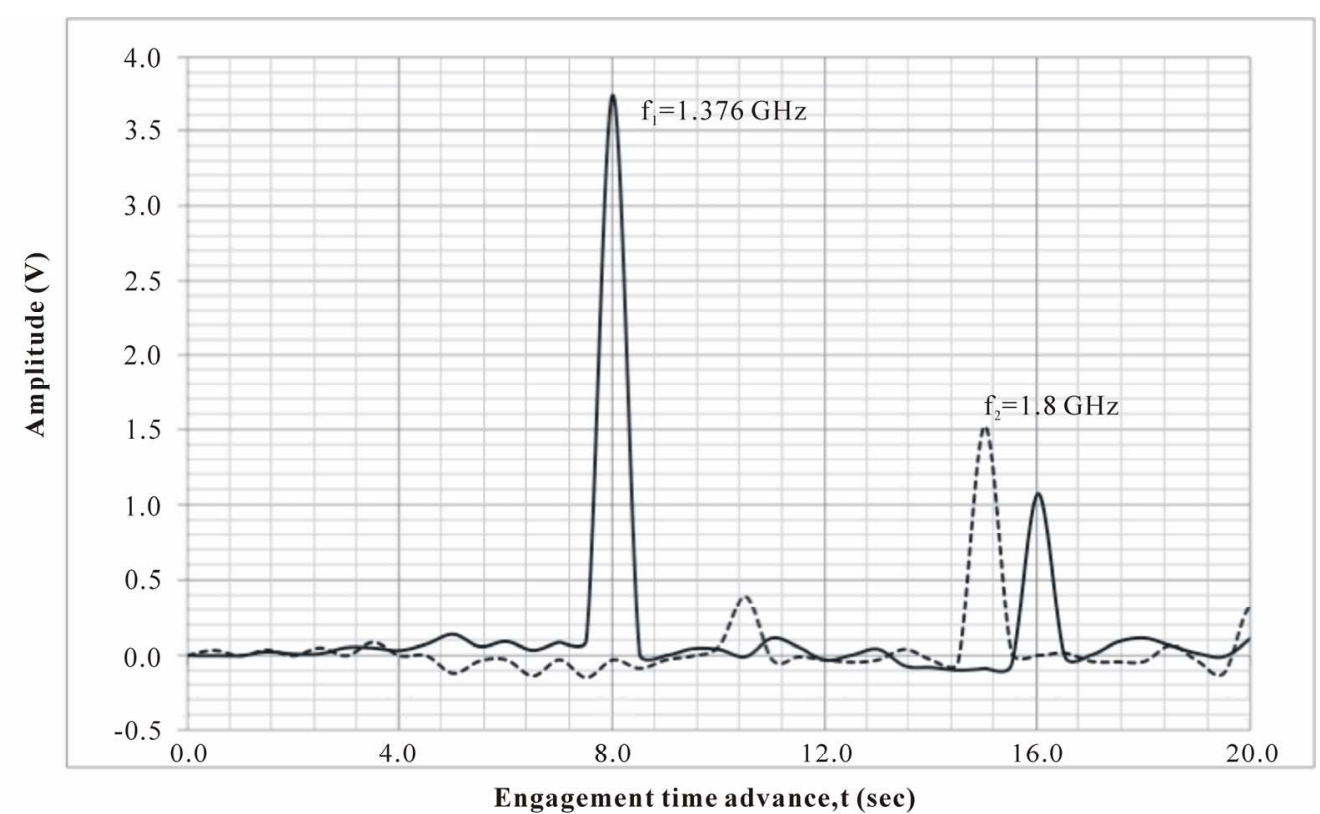

Figure 3. Simulated received response of mobile terminal at typical S-PCN frequencies.

minimizing error $\varepsilon(t)$ at each time index:

$$
\varepsilon(t)=Y(t)-A \hat{U}(t)
$$

Ensuring fast convergence a local minimum is sought leading to establishing threshold value; i.e.,

$$
\xi(t)=\sum_{j=0}^{k}\left(\beta_{t}(j)-\beta_{t-1}(j)\right) \leq \alpha_{t h}
$$

where $\alpha_{t h}$ is the local minimum threshold. The adaptation gain $g(m)$ is introduced for coefficient updating recursion for the period of the signal measurement:

$$
g(m)=\left(\sum_{i=0}^{m} \frac{\hat{U}(i)}{X(i)+N(i)}\right)
$$

where $m$ is the period which the mobile terminal engages the network.

As shown in Figure 3, application of adaptive filter in conjunction with the antenna array processing shows encouraging responses in terms of interference reduction. The implication of our method is that the variation of the filter's weights, as a result of movement of the mobile users, may affect the effectiveness of the system as the satellite moves from orbit to another; low earth orbit (LEO) through to geostationary orbit (GEO), for example. However, a mobile user of any of the systems might experience the same problem since the environment is changing too rapidly for the weights to converge. This is an area that is being investigated. Also, the relative motion between the user and the satellite reference may be another source of error resulting in Doppler shift. Recent work of [16] suggests that using frequency lock loop could compensate for this error. Whilst our technique has utilised uniform weight for the antenna array, this should not a problem if variable weights were to be utilised [17].

\section{Conclusions}

The inherent communication capabilities of satellites render them an attractive solution for the personal communication in mountainous terrain. The paper has presented an antenna-array plus adaptive filtering model as a way of processing multipath signals in satellite personal communication systems. This technique has shown that it can eliminate an uncorrelated signals residing in the antenna sidelobes successfully. This technique is easily adaptable to S-PCN in LEO operational environment as a result of shorter time required by LEO satellites to move across the sky.

\section{References}

[1] L. Ghedia, "Satellite Personal Communication Networks," In: B. G. Evans Ed., Satellite communication systems, Exeter: Institution of Electrical Engineers, 2008.

[2] R. Conte, "Satellite Rural Communications: Telephony and Narrowband Networks," International Journal of Satellite Communications and Networking, Vol. 23, No. 5, 2005, pp. 307-321.

[3] R. Septiawan, "Multiservice Traffic Allocation in LEO Satellite Communications," Bond University, Australia, 2004.

[4] P. F. Driessen, "Prediction of Multipath Delay Profiles in Mountainous Terrain," IEEE Journal on Selected Areas 
in Communications, Vol. 18, No. 3, 2000, pp. 336-346.

[5] J. Marais, "Satellite Propagation Analysis in a Masking Environment for GNSS Application," IEEE Vehicular Technology Conference, Vol. 6, 2005, pp. 4066-4070.

[6] M. B. Pursley, "Direct-Sequence Spread-Spectrum Communication for Multipath Channels," IEEE Transactions on Microwave Theory and Techniques, Vol. 50, No. 3, pp. 653-660.

[7] M. O. Kolawole, "Satellite Communication Engineering," Marcel Dekker, New York, 2002.

[8] M. Zeng, A. Annamalai and V. K. Bhargava, "Recent Advances in Cellular Wireless Communications," IEEE Communication Magazine, Vol. 37, No. 9, 1999, 128138.

[9] A. Benella, J. Berkebile and A. Jacomb-Hood, "A TwoElement Phased-Array Antenna for Reducing Multipath Effects in Handheld SATCOM Units," Microwave Journal, Vol. 8, No. 1, 1996, pp. 22-36.

[10] M. I. Skolnik, "Introduction to Radar Systems," McGraw-Hill, Singapore, 1981.

[11] M. O. Kolawole, "Radar Systems, Peak Detection and Tracking," Elsevier, Oxford, 2003.
[12] E. Lutz, "Issues in Satellite Personal Communication Systems," Wireless Networks, Vol. 4, No. 2, 1998, pp. 109-124.

[13] M. O. Kolawole, "A Course in Telecommunication Engineering," S Chand, New Delhi, 2009.

[14] M. A. Ingram, R. Romanofsky, R. Q. Lee, F. Miranda, Z. Popovic, J. Langley, W.C. Barott, M. U. Ahmed and D. Mandi, "Optimizing Satellite Communications with Adaptive and Phased Array Antennas," Earth Science and Technology Conference, USA, 2004, pp. 1-7.

[15] K. F. Akingbade and M. O. Kolawole, "Utilizing Antenna Array Concept as Source Signal to Modeling Artefacts in Electrocardiogram Signals," Journal of Communication and Computer, Vol. 7, No. 9, 2010, pp. 67-70.

[16] M. Rao, L. L. Presti, M. Fantino and G. Garbo, "A Software Receiver Adaptive Phase Lock Loop Method," International Global Navigation Satellite Systems Society (IGNSS) Symposium, December 2009, pp. 1-13.

[17] S. E. Iwasokun, "Elimination of Interference and Multipath in Satellite Personal Communication Systems Using Adaptive Antenna Array," The Federal University of Technology Akure, Nigeria, 2010. 